$45 \mid 2010$

Langue française, identité(s) et école(s) : le cas de la minorité catholique au Levant (milieu XIX ${ }^{e}-X^{e}$ siècles). Représentations identitaires et apprentissage des langues : Europe, bassin méditerranéen ( $\left.\mathrm{XV}\right|^{\mathrm{e}}-\mathrm{XX}{ }^{\mathrm{e}}$ siècles)

\title{
Raymond Renard. Structuro-global et verbo-tonal. Variations 1962-2010. Essais de didactique des langues.
} Mons : Éditions du CIPA, 2010, 332 p., (<http://w3.umh.ac.be/ cipa/> ; et pour toute commande : <CIPA@umh.ac.be>).

Michel Berré

\section{(2) OpenEdition}

Édition électronique

URL : https://journals.openedition.org/dhfles/2497

DOI : $10.4000 /$ dhfles. 2497

ISSN : 2221-4038

\section{Éditeur}

Société Internationale pour l'Histoire du Français Langue Étrangère ou Seconde

Édition imprimée

Date de publication : 1 juin 2010

Pagination : 254-260

ISSN : 0992-7654

Référence électronique

Michel Berré, «Raymond Renard. Structuro-global et verbo-tonal. Variations 1962-2010. Essais de didactique des langues. ", Documents pour l'histoire du français langue étrangère ou seconde [En ligne], 45 | 2010, mis en ligne le 31 août 2013, consulté le 27 mai 2021. URL : http://journals.openedition.org/ dhfles/2497 ; DOI : https://doi.org/10.4000/dhfles.2497

Ce document a été généré automatiquement le 27 mai 2021.

(c) SIHFLES 


\section{Raymond Renard. Structuro-global et verbo-tonal. Variations 1962-2010. Essais de didactique des langues.} Mons : Éditions du CIPA, 2010, 332 p., (<http://w3.umh.ac.be/ cipa/> ; et pour toute commande :<CIPA@umh.ac.be>).

\section{Michel Berré}

1 Depuis les années quatre-vingt-dix, les membres de l'association SGAV (structuroglobal audio-visuel) ont publié une série d'ouvrages dont l'objectif est de mieux faire connaître le SGAV, "méthode» apparue au cœur des années cinquante, dans la mouvance de la naissance des didactiques disciplinaires, et qui a profondément marqué l'histoire de l'enseignement des langues dans la seconde moitié du XXe siècle. Ils entendent répondre ainsi à ce qu'ils appellent eux-mêmes un «déficit de communication ", les "Sgavistes » s'étant pendant de nombreuses années plutôt préoccupés de l'élaboration de supports pédagogiques et de formation des maitres que de la consolidation théorique et de l'étayage de leurs choix, intuitions et pratiques méthodologiques. Signalons parmi ces publications les quatre tomes de synthèse intitulés Apprentissage d'une langue étrangère/seconde parus chez De Boeck ${ }^{1}$ et respectivement édités par De Vriendt (2000), Renard (2002), Rivenc (2003) et Barbé Courtillon (2005).

2 L'ouvrage publié aujourd'hui par Raymond Renard s'inscrit dans cette politique de communication. «Il y a un temps [et] un âge pour tout », rappelle l'auteur (p. 237)... Le temps du toujours très actif octogénaire n'est plus à l'élaboration de nouvelles recherches, mais à la compréhension, à la reformulation, à la transmission de ce qui a été dit et fait pendant près d'un demi-siècle en essayant de situer cette "rechercheaction » dans une doctrine elle-même en constante évolution et en relation avec la réalité du terrain, seule « libératrice, comme le souligne l'auteur, de la doxa » (p. 7). Impossible en quelques lignes de revenir sur l'ensemble du parcours professionnel de l'auteur dont toute la carrière s'est déroulée à l'université de Mons (fondateurdirecteur de l'École d'interprètes internationaux - aujourd'hui Faculté de traduction et 
d'interprétation ; fondateur du Centre interfacultaire de phonétique appliquée (CIPA) aujourd'hui Centre international de phonétique appliquée; fondateur-président de l'Institut de linguistique - aujourd'hui Institut des sciences du langage; doyen de la Faculté des sciences psychopédagogiques; vice-recteur; coordinateur de la Chaire UNESCO en aménagement linguistique et didactique des langues, etc.) ou sur ses très nombreuses publications. Le lecteur intéressé se reportera avec profit au volume d'hommage publié en 1991, intitulé Mélanges de phonétique et didactique des langues. Hommage au professeur Raymond Renard (cf. Landercy A., éd. 1991). Nous nous contenterons de présenter la structure de l'ouvrage de Renard (a) et, dans un second temps, de mettre en parallèle les évolutions terminologiques de ce qui s'appelait dans les années soixante la "méthode Zagreb/Saint-Cloud» (ou la méthode «Guberina/ Rivenc »), avec les profondes transformations qui ont modifié le domaine du français langue étrangère et plus généralement celui de la didactique des langues depuis la Deuxième Guerre mondiale (b).

4 (a) L'ouvrage de 2010 reprend le plan et le contenu de celui de 1993 (Variations sur la problématique SGAV: essais de didactique des langues, université de Mons, Éditions du CIPA). La nouveauté est constituée par l'insertion d'extraits tirés des articles et des ouvrages publiés par Renard depuis cette date. L'ordre de présentation des textes est chronologique à l'intérieur de trois sections (déjà présentes en 1993) :1) les fondements théoriques ; 2) le rôle de la phonétique ; 3) la didactique et la formation des maîtres (p.9). On notera le rapprochement du terme didactique avec le domaine de la formation professionnelle.

5 L'objectif du volume est de «contribuer à une meilleure connaissance du mouvement SGAV» (p.9). Il est constitué de la reproduction d'un certain nombre d'extraits d'articles ou d'ouvrages (41 au total) publiés par Renard entre 1962 et 2010. Ces extraits sont présentés sous leur forme originale ; toutefois dans un souci d'économie, les notes de bas de page n'ont été conservées que lorsqu'elles ont été jugées utiles; de même pour les références bibliographiques toutes regroupées en fin de volume. Enfin, les points de suspension entre crochets indiquent les coupures effectuées par l'auteur essentiellement, nous a-t-il confié, pour éviter les redites.

Un coup d'œil sur la table des matières permet de se rendre compte de l'intense activité de l'auteur, que ce soit pour souligner la longévité du «mouvement SGAV » (ce qu'il appelle le "défi de la durée », p. 303-309, texte écrit en 2005), la nécessité de ne pas couper l'enseignement des langues d'une réflexion plus globale sur les valeurs éducatives (la laïcité, la paix..., p. 295-302, texte daté de 2000) ou encore l'indispensable prise en compte des cultures et contextes éducatifs, en particulier en Afrique (p. 137-142, texte de 2000). Bref, une approche largement humaniste de l'éducation linguistique sachant aussi témoigner d'une profonde humanité quand il s'agit de rendre hommage à la figure de l'un des fondateurs de «mouvement SGAV » - devenu l'ami d'une formidable aventure - Petar Guberina disparu en 2005 (p. 231-236, texte daté de 2006).

(b) Mouvement, méthode, méthodologie, problématique, hypothèse... L'évolution des termes qui depuis cinquante ans ont servi de support au qualifiant «SGAV » nous paraît révélatrice des transformations qui ont affecté la didactique des langues, domaine encore considéré comme relevant essentiellement de la formation professionnelle au lendemain de la Seconde Guerre mondiale et qui, progressivement, 
est devenu une discipline universitaire générant ses propres concepts et ses propres recherches.

8 Le premier terme à relever est celui de "méthode » dont Renard s'est servi en 1962 pour désigner l'objet nouveau devant lequel il se trouve quand il découvre Voix et Images de France, manuel d'enseignement du français aux étrangers, conçu par l'équipe du CREDIF (Centre de recherche et d'étude pour la diffusion du français) - dirigée par Paul Rivenc (École normale supérieure de Saint-Cloud) - et par l'Institut de phonétique de l'université de Zagreb, piloté par Petar Guberina. Au départ, l'usage que fait Renard du terme "méthode » est assez indifférencié ; il sert tantôt à désigner les hypothèses relatives aux langues et à leur appropriation (essentiellement issues des travaux de Guberina sur les pathologies du langage), tantôt à nommer ce que l'on appelle aussi «manuel».

Dans un second temps, la paire "méthode/méthodologie " apparaît sous la plume de Renard; le premier terme se spécialise alors pour désigner le niveau des manuels (notamment ceux conçus par le CREDIF pour l'enseignement du français comme langue étrangère), le second est réservé à celui des hypothèses relatives à la manière d'enseigner/ apprendre une langue. C'est dans ce sens que l'on a parlé (et que l'on parle encore) de "méthodologie SGAV » (cf. le colloque SGAV à l'université de Mons en 1976 et ses deux volets : « Méthodologie SGAV et système verbo-tonal : tendances nouvelles » et «La méthodologie SGAV face au problème du Niveau-Seuil »; nous soulignons).

$\mathrm{Au}$ cours des années soixante-dix, le syntagme "méthodologie SGAV » a progressivement été concurrencé par celui de "problématique SGAV». La première attestation relevée sous la plume de Renard date de 1974 et associe nettement « méthodologie » et " problématique » : «Finalement, la méthodologie SGAV m'apparaitt comme la problématique la plus apte à justifier l'existence même de l'école [...] » (250 ; nous soulignons). Le colloque SGAV de 1981, intitulé « Problématique SGAV et approche communicative » a consacré cette nouvelle orientation. Rivenc (2003) - par ailleurs organisateur du colloque de 1981 - traite de cette question spécifique dans une partie du chapitre consacré à l'histoire du mouvement SGAV, "D'une 'méthodologie' à une 'problématique' SGAV » (cf. Rivenc 2003, p. 120-125).

11 Cette évolution terminologique s'est faite dans un contexte plus difficile pour le mouvement SGAV qui, après le succès des années soixante, a affronté la décennie suivante de nombreuses critiques venant d'autres courants méthodologiques mais aussi, plus radicalement, de didacticiens proclamant la fin des «méthod(ologi)es». Le terme "problématique » constitue en premier lieu une réponse à cette contestation en positionnant le SGAV comme un «lieu de débats " plutôt que comme un organe de diffusion de certitudes méthodologiques. Mais ce changement de terme cherche aussi c'est du moins notre interprétation - à intégrer le mouvement SGAV au processus d'universitarisation dans lequel s'inscrit progressivement le français langue étrangère (et la didactique des langues), en particulier dans les années quatre-vingt. Désormais, il s'agit moins d'apporter des réponses à une demande de formation des enseignants (sous la forme de manuels ou de stages, souvent conçus comme une préparation à l'usage de ces manuels) que de formuler de nouvelles questions auxquelles des recherches scientifiques pourront apporter des réponses à l'instar de ce qui se fait dans d'autres disciplines institutionnellement reconnues au plan universitaire. Dans cette perspective, le terme " problématique » (défini par le Trésor de la langue française comme l'« ensemble des problèmes élaborés par une science donnée et considérés comme 
délimitant le domaine qui lui est propre ») convient évidemment mieux que celui de méthode ou de méthodologie. Le peu de relais des "Sgavistes » au sein des universités (notamment françaises) explique sans doute la difficulté éprouvée par le mouvement SGAV à se positionner sur ce terrain nouveau et à effectuer une véritable « conversion ".

Même si d'autres noms ont accompagné le sigle SGAV (« hypothèse ", "optique », ou encore « approche »), le terme " problématique » s'est maintenu jusqu'à aujourd'hui. Il est utilisé par Renard dans le titre de son ouvrage de 1993 (cf. supra), mais est absent de celui de 2010. Il figure cependant dans les quatre mots-clés retenus par Renard dans son introduction (à côté de structuro-global, verbo-tonal et parole), tout en étant dépouillé de son sigle qualifiant (SGAV).

Deuxième évolution donc - et non des moindres - celle qui a conduit Renard à abandonner dans son titre le sigle SGAV au profit de verbo-tonal.

Le mouvement SGAV est apparu vers 1955-1956 sans faire l'objet d'une dénomination « officielle»; selon Rivenc, les adjectifs « audio-visuel » et «structuro-global » ont été choisis en 1961 sur proposition de Guberina qui employait ce terme; par la suite, le mouvement s'est constitué en association en 1975, devenue internationale en 1978 (cf. Revue de Phonétique Appliquée 1981, n 59-60, p. 215-222). Dans son premier texte qui concerne Voix et Images de France en 1962, Renard parle uniquement de "méthode audio-visuelle ». "Structuro-global » arrive un an plus tard, dans le titre d'un article plus long publié dans le Bulletin scientifique de l'Institut supérieur du Commerce de la Province de Hainaut (Belgique): "Une révolution dans l'enseignement des langues vivantes: la méthode audio-visuelle et structuro-globale de Saint-Cloud - Zagreb" (1963 ; nous soulignons). On notera l'ordre (la méthode est d'abord audio-visuelle avant d'être structuro-globale), le sigle AVSG ayant précédé celui de SGAV avant d'être concurrencé puis progressivement supplanté. Le titre de l'article qui ouvre le premier numéro de la Revue de Phonétique Appliquée (fondée par Renard en 1965) est explicite à cet égard : « La Méthode audio-visuelle structuro-globale » par Guberina.

La généralisation du sigle SGAV serait effective vers 1968 (Rivenc, op. cit.); progressivement, le qualifiant « audio-visuel » est passé au second rang, toute l'activité des concepteurs du SGAV se portant sur la définition à donner aux termes "structure " et « global ». Le deuxième colloque international de l'association SGAV (Mons, 1971) a consacré l'un de ses trois thèmes de réflexion à cette question (« Vers une définition de l'expression 'structuro-globale' (sic) », actes parus dans la Revue de Phonétique Appliquée, $\left.\mathrm{n}^{\circ} 21,1972\right)$.

À partir de cette date (et sans doute pour se distinguer d'autres cours audio-visuels ne correspondant pas aux options des concepteurs de Voix et Images de France), le terme " audio-visuel »- simple référence aux moyens techniques permettant de transposer artificiellement en classe la nature structuro-globale de la communication orale - passe au second rang; une évolution qui conduit Renard en 2010 à supprimer le terme puisqu'il parle désormais d'" approche structuro-globale» et de "processus verbotonal », le sigle SGAV ayant disparu du titre. L'on notera que dans la table des matières de l'ouvrage de Renard - qui reprend les titres originaux des textes rédigés entre 1962 et 2010 - l'adjectif «audio-visuel» est absent (sous une forme explicite) alors que le terme « structuro-global » apparaît au moins un dizaine de fois.

17 En substituant dans son titre "verbo-tonal» à "audio-visuel ", Renard entend souligner, selon ses propres termes, «le caractère consubstantiel de l'approche 
structuro-globale et du processus verbo-tonal dans l'enseignement/apprentissage de la parole » (p. 7). Il rejoint ainsi, nous semble-t-il, les préoccupations de Jacques Cortès qui, dans un célèbre article de 1981 («L'ancien et le nouveau testament de la didactique des langues ", paru dans la Revue de Phonétique Appliquée, n59-60) déplorait «que la plupart des historiens contemporains de l'approche SGAV om[issent] même d'évoquer le nom de Guberina et l'apport essentiel de sa Théorie Verbo-Tonale » (p. 253) ${ }^{2}$.

Ce faisant, Renard réaffirme les fondements psycholinguistiques du "mouvement SGAV ", la notion de structuro-global concernant à la fois le fonctionnement du cerveau et l'organisation de la langue ; mais à cette composante biologique, il convient d'ajouter une dimension sociale, Guberina ayant toujours conçu la parole non pas comme un phénomène individuel (Saussure), mais comme un acte social (Bally) tirant sa valeur de l'ensemble des paramètres de la situation de communication ; cela apparait très clairement dans la définition que le phonéticien de Zagreb donne du terme "structure »: "La structure dans le SGAV [...] est conçue sur la base des rapports réciproques et permanents entre société et individu » (Guberina, cité dans D. Coste éd., Aspects d'une politique de diffusion du français langue étrangère. Matériaux pour une histoire, Paris : Hatier, 1984, p. 90).

Dans cette perspective, la "méthode SGAV » pourrait être redéfinie comme une «hypothèse d'ordre psycho- socio-linguistique » sur l'apprentissage de la parole (en ce compris la langue écrite et le langage intérieur); ce qui ouvre la porte à bien des recherches sur la manière d'articuler les composantes biologiques et culturelles dans les travaux relevant du domaine (cf. l'article "L'homme et son langage », p. 143-145, daté de 2004).

L'ouvrage de Renard permet au lecteur de s'immerger dans près de cinquante années du « passé » de la didactique des langues. Le trop bref balisage que nous avons proposé, fondé sur l'usage de certains termes par Renard - usage qui n'est bien entendu que partiellement idiosyncratique - n'est qu'un parcours de lecture parmi bien d'autres possibles. Didacticiens et historiens de l'enseignement des langues trouveront dans l'ouvrage de Renard une source précieuse de renseignements ; mais ils n'oublieront pas toutefois d'aller consulter les textes originaux, ne fût-ce que pour prendre la mesure des contextes de production et de réception de ces textes et pour les rétablir dans leur version intégrale et originale.

\section{NOTES}

1. <http://www.deboeck.com/>

2. Rappelons que la thèse de Guberina, soutenue à la Sorbonne en 1939, a été rééditée par le Centre international de phonétique appliquée (CIPA) en 1993: Valeur logique et valeur stylistique des propositions complexes : théorie générale et application au français (il s'agit de la réimpression de la $2^{\mathrm{e}}$ édition parue à Zagreb aux éditions Epoha en 1954). Sur Guberina, l'on consultera Claude Roberge (2003, éd.) Rétrospection. Petar Guberina, Zagreb, Artresor Naklada, 2003 et M. Berré (2004, 
éd.), Linguistique de la parole et apprentissage des langues. Questions autour de la méthode verbo-tonale de P. Guberina, Mons : Éditions du CIPA.

\section{AUTEUR}

MICHEL BERRÉ

Université de Mons 